\title{
The Ubiquitin-Proteasome Proteolytic System
}

From Classical Biochemistry to Human Diseases 
This page is intentionally left blank

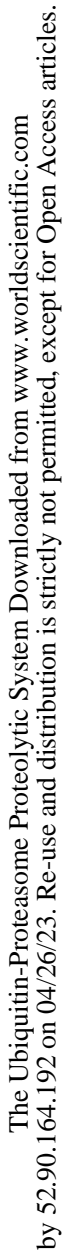


Recent Advances in Human Biology - Volume

Series editor: Charles E. Oxnard

Centre for Human Biology

The University of Western Australia

\section{The Ubiquitin-Proteasome Proteolytic System From Classical Biochemistry to Human Diseases}

\section{Editors}

\section{Aaron J. Ciechanover}

Bruce Rappaport Faculty of Medicine

Technion-Israel Institute of Technology, Haifa, Israel

\section{Maria G. Masucci}

Microbiology and Tumor Biology Center, Karolinska Institutet, Stockholm, Sweden 
Published by

World Scientific Publishing Co. Pte. Ltd.

P O Box 128, Farrer Road, Singapore 912805

USA office: Suite 1B, 1060 Main Street, River Edge, NJ 07661

UK office: 57 Shelton Street, Covent Garden, London WC2H 9HE

\section{British Library Cataloguing-in-Publication Data}

A catalogue record for this book is available from the British Library.

\section{THE UBIQUITIN-PROTEASOME PROTEOLYTIC SYSTEM}

Copyright $@ 2002$ by World Scientific Publishing Co. Pte. Ltd.

All rights reserved. This book, or parts thereof, may not be reproduced in any form or by any means, electronic or mechanical, including photocopying, recording or any information storage and retrieval system now known or to be invented, without written permission from the Publisher.

For photocopying of material in this volume, please pay a copying fee through the Copyright Clearance Center, Inc., 222 Rosewood Drive, Danvers, MA 01923, USA. In this case permission to photocopy is not required from the publisher.

ISBN 981-238-100-7 


\section{Preface}

The finding that cellular proteins are turning over-synthesized and degraded constantly-has traversed a torturous road from its discovery in the 1940s until it has reached its current central position as a major regulatory pathway. The dynamic state of the proteome was discovered by Rudolph Schoenheimer who used radiolabeled compounds to demonstrate that proteins are in a constant state of generation and destruction (1). Yet, the extent, the mechanisms and the physiological significance of roles of protein degradation have remained elusive for many years. Simpson reported that degradation of labeled proteins in liver slices requires metabolic energy (2). This - thermodynamically paradoxical findingwhere investment of energy is still required for the degradation of energyrich macromolecules-proteins-to low energy small molecules-amino acids-has been corroborated in many studies ever since in both eukaryotes and prokaryotes. Since proteolysis is an exergonic process, the requirement for energy had remained an enigma. Simpson tried to explain that "The fact that a supply of energy seems to be necessary for both the incorporation and the release of amino acids from protein might well mean that the two processes are interrelated". He concluded however by saying that "...the fact that protein hydrolysis as catalyzed by the familiar proteases and peptidases occurs exergonically, together with the consideration that autolysis in excised organs or tissue minces continues for weeks, long after phosphorylation or oxidation ceased, renders improbable the hypothesis of the direct energy dependence of the reactions leading to protein breakdown" (2). The basic principle that cleavage of a peptide bond is exergonic, has not and could not have been challenged. Yet, the simple notion that proteases cannot exist in one compartment with their substrates without an energy barrier separating them, along with the high specificity of the process as we currently know it, makes energy investment in an 
apparently exergonic process something we all accept now. Yet, the road to this acceptance has been long.

The discovery of the lysosome by Dr. Christian de Duve (reviewed in Ref. 3) has resolved some of the enigmas. Since the lysosome contains many acidic proteases, it was suggested that it must play a role in the degradation of intracellular proteins. Mortimore demonstrated a direct correlation between accelerated protein degradation that follows deprivation of nutrients to perfused liver, and increased lysosomal autophagy that is accompanied by a variety of structural alterations in the lysosomal system. Both the accelerated degradation and the structural changes could be reversed by re-supplementation of amino acids and hormones or serum (see for example Ref. 4). It was found that energy is required for activity of the lysosomal membrane proton pump that maintains the low intralysosomal $\mathrm{pH}$ necessary for optimal activity of the proteases (5). Different lines of experimental evidence along with the development of specific inhibitors strongly suggested that multiple pathways are involved in intracellular protein degradation, and the lysosome plays a role only in certain aspects of this process. Proteins were classified into short- and long-lived (reviewed in Refs. 6,7), but their different stability could not be explained based on the known mechanism of action of the lysosome that involves micro- and macroautophagy. During this process, entire droplets of cytosol and even subcellular organelles are engulfed with all the contained proteins digested at similar rates. While it was clear that lysosomal proteases are neither selective nor specific, certain studies still attempted to attribute specificity to lysosomal degradation. According to one model for example, all cellular proteins are engulfed into the lysosome, but only short- lived proteins that are sensitive to lysosomal proteases are degraded, whereas the more resistant, long-lived proteins escape back into the cytosol (8). The development of specific inhibitors of lysosomal proteases and of lysosomotropic agents-weak bases such as chloroquine or ammonium chloride - that are entrapped within the lysosome and increase the $\mathrm{pH}$, thus inactivating lysosomal proteases-enabled researchers to examine in more detail the existence of distinct proteolytic pathways. Knowles and Ballard (9) and Neff and colleagues (10) demonstrated that leupeptin, antipain and chymostatin-specific lysosomal protease inhibitors--inhibit selectively degradation of long-lived but not of short-lived and abnormal proteins. 
Poole and colleagues demonstrated that the lysosomotropic agent chloroquine selectively inhibits enhanced protein breakdown induced in cultured cells by depletion of serum, but has no effect on the degradation of cellular proteins under basal metabolic conditions (11). In an extremely elegant experiment, yet ingenious in its simplicity, he showed that chloroquine does not inhibit the degradation of endogenous cellular proteins that were metabolically labeled with ${ }^{3} \mathrm{H}$-leucine, but at the same time and in the same ${ }^{3} \mathrm{H}$-leucine-labeled cells, strongly inhibits the degradation of either endocytosed BSA, or endocytosed soluble cellular proteins that were prepared from identical cells metabolically labeled with ${ }^{14} \mathrm{C}$-leucine (11). $\mathrm{He}$ concluded that intracellular proteins degraded under stress, or endocytosed/pinocytosed extracellular proteins are degraded within lysosomes following their engulfment from the cytosol or transfer from the cell membrane to the lysosome along the vacuolar system, respectively. In contrast, under basal metabolic conditions, intracellular proteins, and in particular short-lived ones, are degraded by a yet unidentified nonlysosomal system(s) (reviewed in Refs. 7 and 12).

To identify and characterize this non-lysosomal system, Etlinger and Goldberg chose the reticulocyte as a model system. This cell lacks lysosomes and is involved in extensive degradation of its organelles and enzymatic systems prior to maturation in the bone marrow and conversion to a circulating erythrocyte. They found that the reticulocyte contains an ATPdependent proteolytic system that degrades abnormal, amino acid analogcontaining, short-lived proteins (13). Working in parallel, Hershko and Ciechanover fractionated the reticulocyte extract and found that a small$\sim 8.0 \mathrm{kDa}$-heat stable protein is necessary to reconstitute proteolysis of a model substrate in a crude lysate from which it was first removed during fractionation (14). The protein was designated $\underline{A}$ TP-dependent $\underline{P}$ roteolysis $\underline{F}$ actor- 1 (APF-1), as it became clear that the system contains several additional factors that may act in concert. Mechanistic studies revealed that multiple moieties of APF-1 are covalently conjugated-in an ATPdependent mode-to the substrate (15). This surprising finding led the two researchers, along with Rose, to propose a model according to which degradation of a protein via the system involves two steps (i) conjugation of multiple molecules of APF-1 to the substrate, and (ii) degradation of the tagged substrate with release of reusable APF-1 (16). Parallel studies 
identified APF-1 as ubiquitin, a known protein of hitherto unknown function $(17,18)$. Ubiquitin was discovered by Goldstein and colleagues as a protein that induces differentiation of $\mathrm{B}$ and $\mathrm{T}$ cells, and is ubiquitously distributed in prokaryotes and eukaryotes, hence its name (19). Later analyses revealed that it is not involved in regulating lymphocytes development, and that prokaryotes do not express it. Yet, the descriptive name was retained. An interesting finding related to ubiquitin was the identification by Busch and colleagues of the nucleolar protein A24 (see for example Ref. 20). Structural analyses $(21,22)$ revealed that $A 24$ has a unique bifurcated structure in which ubiquitin is conjugated-in an isopeptide bond-via its C-terminal $\mathrm{Gly}^{76}$ to the $\varepsilon-\mathrm{NH}_{2}$ group of Lys ${ }^{119}$ of histone $2 \mathrm{~A}$. The function of protein A24 has remained an unsolved mystery to our days. Yet, the finding that its level is decreased following hydrolysis to its two components, histone $\mathrm{H} 2 \mathrm{~A}$ and ubiquitin, during liver regeneration (23) or erythropoesis (24), as well as a later finding that it is associated preferentially to nucleosomes that are localized at the 5' end of actively transcribed genes (25), led to the hypothesis that it plays a role in transcriptional regulation. As noted, all the changes observed in the level of the protein involve its hydrolysis and reassembly and not degradation and resynthesis. In light of our current understanding of the ubiquitin system, this is because proteins that are modified by a single moiety of ubiquitin cannot be recognized by the $26 \mathrm{~S}$ proteasome, the protease of the system (see below) that degrades only multiply ubiquitinated substrates. Identification of APF-1 as ubiquitin and the known structure of A24 led to the hypothesis that the C-terminal Gly residue of ubiquitin must be activated prior to its conjugation in a mechanism that is enzymatically similar to the activation of amino acids by aminoacyl tRNA-synthetase during ribosome-based polypeptide synthesis, or to the activation of amino acids during ribosome-free oligopeptide biosynthesis (see for example Ref. 26). Indeed, experiments in fractionated extracts showed that intermediates similar to those generated during amino acid activation, are generated also during activation of ubiquitin (27). Using the deciphered activation of ubiquitin, Ciechnaover and Hershko used immobilized ubiquitin to purify, via mechanism-based "covalent" affinity chromatography and reversal of the activation reaction, the first enzyme in the ubiquitin pathway cascade, the ubiquitin-activating enzyme, E1 (28). Purification of the two other enzymes in the ubiquitin relay reaction, the 
ubiquitin-carrier protein, E2 (later designated also the ubiquitin-conjugating enzyme, UBC), and the ubiquitin-protein ligase, E3 (29) followed shortly after. The many members of the E3 family bind the target substrates via defined motifs and endow the system with its high specificity. Generation of antibodies to ubiquitin allowed to demonstrate, for the first time, that the system is active also in nucleated cells in vivo and not only in the terminally differentiating reticulocyte-the model cell studied initially: a direct correlation was observed between levels of ubiquitin adducts and rates of abnormal protein degradation induced by incubation of the cells in the presence of amino acid analogues (30). Stronger and more direct evidence was later obtained by Varshavsky, Finley and Ciechanover, who characterized a known cell cycle arrest mutant that loses A24 at the nonpermissive temperature. They identified the mutation as a thermolabile E1 that, when inactive, cannot re-conjugate ubiquitin to histone $2 \mathrm{~A}$ (31). Heat inactivation of the enzyme leads to severe impairment in the degradation of short-lived abnormal proteins generated during incubation of the cells in the presence of amino acids analogs (32). The original observation by Yamada and colleagues that loss of the thermolabile E1 leads to arrest at the S/G2 phase, enabled the three researchers to predict that the ubiquitin system is required for cell cycle progression. This hypothesis was later corroborated by numerous studies demonstrating ubiquitin intermediacy in the degradation of many cell cycle regulators. Later studies led to the discovery that it is a polyubiquitin chain - in which the ubiquitin moieties are linked to one another - that generates the high molecular mass adducts and the proteolytic signal (33). Beforehand, a formal possibility still existed that the high molecular mass adducts represent multiple single moieties attached to distinct lysine residues. The chain is composed of ubiquitin moieties that are linked to one another via an isopeptide bond between the C-terminal Gly76 of one ubiquitin moiety and an internal Lys 48 of the previously conjugated moiety (34). In parallel, the downstream protease-the $26 \mathrm{~S}$ proteasome complex-was discovered (35-37), and the entire pathway could be studied in different experimental systems.

The first insights on the problem of specific substrate recognition began to emerge at that time. Using a biochemical approach, Hershko demonstrated that the $\mathrm{N}$-terminal residue may play a role in recognition of certain model substrates (38), and that recognition is mediated via binding 
of this residue to the ubiquitin ligase (39). Ciechanover showed that tRNAArg is required for the degradation of certain proteins (40) that have acidic N-termini. Arg-tRNA-protein transferase catalyzes a reaction that adds an Arg residue to the acidic $\mathrm{N}$-terminal residue, and the conversion of the charge allows recognition of the substrate by the ubiquitin ligase E3 $\alpha$ (41). Varshavsky used a genetic approach in yeast to generate 20 distinct species of a derivative of $\beta$-galactosidase that differed solely in the identity of the $\mathrm{N}$-terminal residue. Yet, the stability of the proteins varied significantly, which led to the formulation of a rule, the $\mathrm{N}$-end rule, according to which the identity of the N-terminal residue determines the stability of the protein (42). Later studies revealed that the $\mathrm{N}$-terminal recognition signal contains adjacent lysine residues that serve as ubiquitination anchors (43). Recognition of the $\mathrm{N}$-terminal residue cannot provide a general targeting mechanism since the $\mathrm{N}$-terminal residue of most substrates is not accessible for recognition by the ligase as it is acetylated (44). We now know that recognition of proteins by the ubiquitin system is far more complex then originally thought. Proteins are targeted by multiple ligases following recognition of different primary and secondary motifs, post-translational modifications and recognition in trans mediated by association with ancillary proteins. Yet, the discovery of N-terminal recognition, the first targeting motif, was of great importance, as it drew attention to a centrally important problem-the requirement for specific recognition of the substrates.

These early studies did not change the prevailing view that the main role of the system is to rid the cell from mutated/misfolded/abnormal proteins and the research focus remained on the puzzle of how the system selectively recognizes and eliminates abnormal proteins, leaving intact their normal counterparts, when the differences can be minute and sometime indiscernible. It was not until the early $1990 \mathrm{~s}-$ when researchers started to discover that specific key cellular proteins, such as transcriptional and cell cycle regulators, are targeted by the system in a regulated manner (see for example Refs. 45-49) - that we have begun to see an exponential growth in the number of published studies and a general recognition of the role of the system in basic cellular processes such as regulation of transcription, cell cycle progression, growth and differentiation, the immune and inflammatory responses, and quality control. Not too long after that researchers started to 
realize that aberrations in such a complex pathway underlie the pathogenesis of many diseases, both inherited and acquired. The discoveries of ubiquitinlike proteins and their role in non-proteolytic functions such as routing of certain proteins to their subcellular destinations or protecting others from ubiquitination and destruction, of mono-ubiquitination and its role in regulating the endocytic pathway, and of polyubiquitin chains that involve residues other then Lys 48 in transcriptional regulation, have expanded the scope of ubiquitin conjugation beyond degradation and set it in a centrally important position among other regulatory mechanisms. The evolution of two distinct regulatory mechanisms, phosphorylation that is reversible and proteolysis that is irreversible, has been inevitable evolutionarily. For certain processes such as cell cycle progression, the unidirectional movement along a "one way" road must be controlled in a tight manner. Like the wife of Lot that on her way from Gomorrah "looked back from behind him and she became a pillar of salt" (Bible, Torah, Genesis, 19, 26), the cell cycle cannot look or go back.

Where is research on the ubiquitin system heading now? Important knowledge is still missing on the specific function of the E3s and their substrates. It is likely that, based on the recognition of common structural motifs such as the HECT domains, RING fingers and U-boxes, the human genome will unravel hundreds of novel ligases and lead to the discovery of their substrates, the processes involved, and the aberrations caused by selective malfunction of these enzymes. Some of these enzymes will have auto-ubiquitinating activity that may serve as a regulatory, "self-destructive" mechanism, others will have both cis and trans activities. Does BRCA1 have specific substrates? Which processes are derailed by its mutation? These and many other questions still await answers. Resolving the 3D structure of the ligases with their substrate may aid in developing mechanism-based specific drugs that will interfere with specific processes. Protease inhibitors are already making their way as potential drugs against many diseases such as malignancies and immune and inflammatory disorders (see for example Refs. 50, 51), yet they clearly cannot be specific and rely for their activity on a narrow toxicity window. Drugs that target specific ligases will affect a narrower subset of substrates. Better and even more specific drugs may be those that will interfere specifically with the 


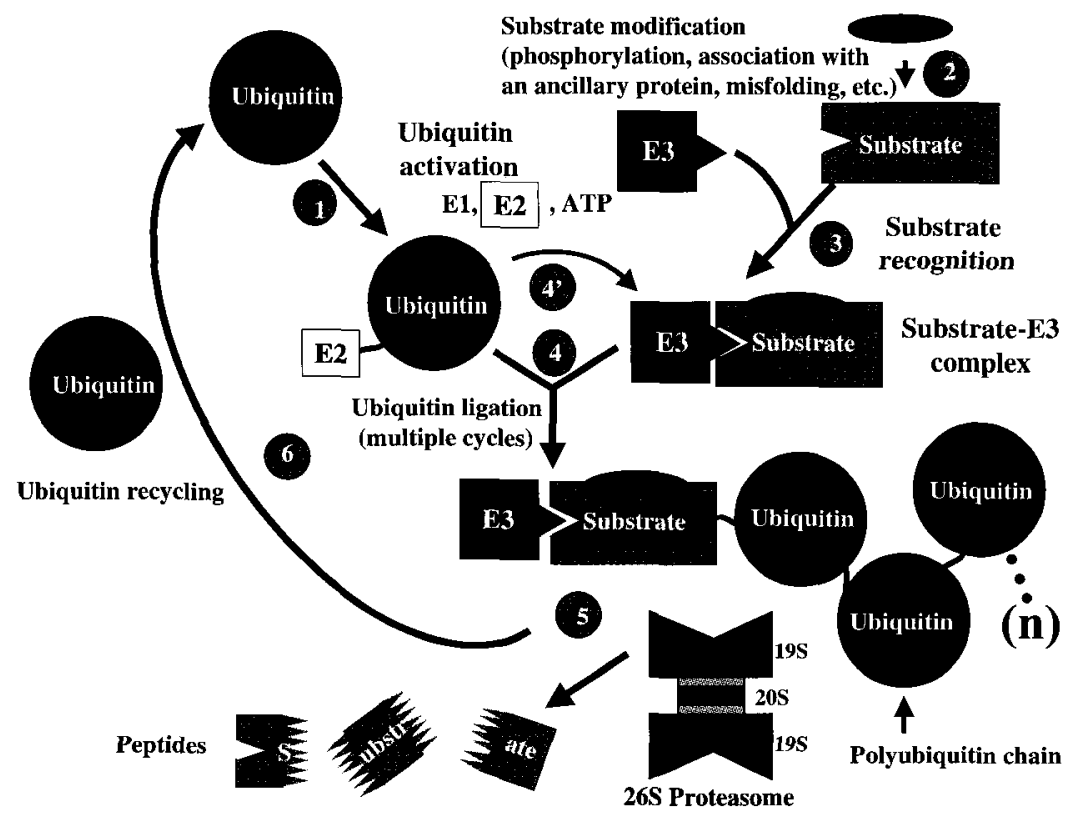

The ubiquitin proteasome pathway. (1) ATP-dependent activation of ubiquitin by the ubiquitin activating enzyme, E1, and by a ubiquitin-carrier protein (ubiquitinconjugating enzyme, UBC), E2, to generate a high-energy E2 ubiquitin intermediate. (2) Modification of the substrate (phosphorylation or oxidation, for example), its association with an ancillary protein (chaperone or a viral protein, for example) or its misfolding are required for its recognition and specific binding to the ubiquitin ligase, E3 (3). (4) Generation of a substrate-anchored polyubiquitin chain catalyzed by direct transfer of the ubiquitin moiety from the E2 ubiquitin complex to the E3-bound substrate (RING finger E3s). (4') Generation of a substrate-anchored polyubiquitin chain catalyzed by transfer of the ubiquitin moiety from the E2 ubiquitin complex to the E3 to generate an additional E3 ubiquitin high energy intermediate from which the activated ubiquitin moiety is transferred to E3-bound substrate [catalyzed by HECT ( $\underline{H}$ omologous to $\underline{E} 6-\mathrm{AP} \underline{\underline{C}}-\underline{T}$ erminus) domain E3s]. U-box-containing E3s have also been described, but their mechanism of function has not been discerned. (5) Degradation of the polyubiquitinated substrate by the $26 \mathrm{~S}$ proteasome complex with release of free and reusable ubiquitin (6) catalyzed by ubiquitin recycling enzymes (ubiquitin C-terminal hydrolases; deubiquitinating enzymes, DUBs; isopeptidases). 
interaction of substrates with ancillary exogenous proteins, such as p53-E6 interaction (52). Interference with endogenous ancillary proteins such as molecular chaperones may prove to be extremely toxic. An additional line of research will involve dissection of non-proteolytic functions of ubiquitin and ubiquitin-like proteins, the requirements for specific substrate recognition and the role of ligases in these processes. All this new knowledge will not only broaden our basic knowledge on the ubiquitin system, but will drive the system from the test tube to the patient bed.

This conference has told us the story of the ubiquitin system, as we currently know it, glowing and shiny. From regulation of basic cellular processes such as cell cycle progression and transcription, through quality control and the pathogenetic mechanisms of disease, from X-ray crystallography of the $26 \mathrm{~S}$ proteasome, to the interaction between substrates and their ligases, to the development of mechanism-based drugs to target specific aberrant processes. But this is the epilogue. It started differently. The history of intracellular protein degradation is an illuminating example of a modern "Cinderella". She started her life in the garbage, literally, helping the cell cleaning it. Carving her way up the mountain, she taught us several important lessons. One is that cleaning garbage is a respected trade. Accumulation of mutated/misfolded/aggregated proteins underlies the pathogenesis of many diseases, including several neurodegenerative disorders such as Huntington's disease. Maintaining the steady state level of growth stimulators, such as $\beta$-catenin and HIF- $1 \alpha$, or tumor suppressors such as p53, is also essential. Accumulation of the first and accelerated degradation of the latter has been implicated in the pathogenesis of several malignancies. Finally, she taught us that normal proteins have to be destroyed as well: programmed destruction of cyclins allows cell cycle progression, whereas removal of transcriptional activators and their inhibitors, regulates specific gene expression. As King Solomon taught us thousands years ago "To everything there is a season and a time to every purpose: A time to be born, and a time to die; a time to plant, and a time to pluck up that which is planted; a time to kill, and a time to heal; a time to break down, and a time to build up" (Bible, Hagiograph, Ecclesiastes, 3, $1-3)$. 


\section{References}

1. Schoenheimer, R. (1942). The Dynamic State of Body Constituents. Harvard University Press, Cambridge, Massachusetts, USA.

2. Simpson, M.V. (1953). The release of labeled amino acids from proteins in liver slices. J. Biol. Chem. 201, 143-154.

3. De Duve, C. (1965). The separation and characterization of sub cellular particles. Harvey Lectures 59, 49-87.

4. Mortimore, G.E., and Mondon, C.E. (1970). Inhibition by insulin of valine turnover in liver. Evidence for a general control of proteolysis. J. Biol. Chem. $245,2375-2383$.

5. Schneider, D.L. (1981). ATP-dependent acidification of intact and disrupted lysosomes: Evidence for an ATP-driven proton pump. J. Biol. Chem. 256, 3858-3864.

6. Schimke, R.T., and Doyle, D. (1971). Control of enzyme levels in animal tissues. Annu. Rev. Biochem. 39, 929-979.

7. Goldberg, A.L., and St. John, A.C. (1976). Intracellular protein degradation in mammalian and bacterial cells. Annu. Rev. Biochem. 45, 747-803.

8. Haider, M., and Segal, H.L. (1972). Some characteristics of the alanineaminotransferase and arginase-inactivating system of lysosomes. Arch. Biochem. Biophys. 148, 228-237.

9. Knowles, S.E., and Ballard, F.J. (1976). Selective control of the degradation of normal and aberrant proteins in Reuber H35 hepatoma cells. Biochem J. 156, $609-617$.

10. Neff, N.T., DeMartino, G.N., and Goldberg, A.L. (1979). The effect of protease inhibitors and decreased temperature on the degradation of different classes of proteins in cultured hepatocytes. J. Cell Physiol. 101, 439-457.

11. Poole, B., Ohkuma, S., and Warburton, M. (1978). Some aspects of the intracellular breakdown of exogenous and endogenous proteins. In: Segal H.L. and Doyle D.J., eds. Protein Turnover and Lysosome Function. Academic Press, New York, NY USA. pp. 43-58.

12. Hershko, A., and Ciechanover, A. (1982). Mechanisms of intracellular protein breakdown. Annu. Rev. Biochem. 51, 335-364.

13. Etlinger, J.D., and Goldberg, A.L. (1977). A soluble ATP-dependent proteolytic system responsible for the degradation of abnormal proteins in reticulocytes. Proc. Natl. Acad. Sci. USA 74, 54-58.

14. Ciechanover A., Hod, Y., and Hershko, A. (1978). A heat-stable polypeptide component of an ATP-dependent proteolytic system from reticulocytes. Biochem. Biophys. Res. Common. 81, 1100-1105. 
15. Ciechanover, A., Heller, H., Elias, S., Haas, A.L., and Hershko, A. (1980). ATP-dependent conjugation of reticulocyte proteins with the polypeptide required for protein degradation. Proc. Natl. Acad. Sci. USA 77, 1365-1368.

16. Hershko, A., Ciechanover, A., Heller, H., Haas, A.L. and Rose, I.A. (1980). Proposed role of ATP in protein breakdown: Conjugation of proteins with multiple chains of the polypeptide of ATP-dependent proteolysis. Proc. Natl. Acad. Sci. USA 77, 1783-1786.

17. Ciechanover, A., Elias, S., Heller, H., Ferber, S. and Hershko, A. (1980). Characterization of the heat-stable polypeptide of the ATP-dependent proteolytic system from reticulocytes. J. Biol. Chem. 255, 7525-7528.

18. Wilkinson, K.D., Urban, M.K., and Haas, A.L. (1980). Ubiquitin is the ATPdependent proteolysis factor I of rabbit reticulocytes. J. Biol. Chem. 255, 7529-7532.

19. Goldstein, G., Scheid, M., Hammerling, U., Schlesinger, D.H., Niall, H.D., and Boyse, E.A. (1975). Isolation of a polypeptide that has lymphocytedifferentiating properties and is probably represented universally in living cells. Proc. Natl. Acad. Sci. USA 72, 11-15.

20. Goldknopf, I.L., Taylor, C.W., Baum, R.M., Yeoman, L.C., Olson, M.O., Prestayko, A.W., and Busch, H. (1975). Isolation and characterization of protein A24, a "histone-like" non-histone chromosomal protein. J. Biol. Chem. 250, 7182-7187.

21. Goldknopf, I.L., and Busch, H. (1977). Isopeptide linkage between nonhistone and histone $2 \mathrm{~A}$ polypeptides of chromosome conjugate-protein A24. Proc. Natl. Acad. Sci. USA 74, 864-868.

22. Hunt, L.T., and Dayhoff, M.O. (1977). Amino-terminal sequence identity of ubiquitin and the nonhistone component of nuclear protein A24. Biochem. Biophys. Res. Commun. 74, 650-655.

23. Ballal, N.R, Kang, Y.J., Olson, M.O., and Busch, H. (1975). Changes in nucleolar proteins and their phosphorylation patterns during liver regeneration. J. Biol. Chem. 250, 5921-5925.

24. Goldknopf, I.L., Wilson, G., Ballal, N.R., and Busch, H. (1980). Chromatin conjugate protein A24 is cleaved and ubiquitin is lost during chicken erythropoiesis. J. Biol. Chem. 255, 10555-10558.

25. Levinger, L., and Varshavsky A. (1982). Selective arrangement of ubiquitinated and D1 protein-containing nucleosomes within the Drosophila genome. Cell 28, 375-385.

26. Lipmann, F., Gevers, W., Kleinkauf, H., and Roskoski, R. Jr. (1971). Polypeptide synthesis on protein templates: the enzymatic synthesis of gramicidin S and tyrocidine. Adv. Enzymol. Relat. Areas Mol. Biol. 35, 1-34. 
27. Ciechanover, A., Heller, H., Etzion-Katz, R. and Hershko, A. (1981). Activation of the Heat-stable Polypeptide of the ATP- dependent Proteolytic System. Proc. Natl. Acad. Sci. USA 78, 761-765.

28. Ciechanover, A., Elias, S., Heller, H. and Hershko, A. (1982). "Covalent affinity" purification of ubiquitin activating enzyme. J. Biol. Chem. 257, $2537-2542$.

29. Hershko, A., Heller, H., Elias, S. and Ciechanover, A. (1983). Components of ubiquitin-protein ligase system: Resolution, affinity purification and role in protein breakdown. J. Biol. Chem. 258, 8206-8214.

30. Hershko, A., Eytan, E., Ciechanover, A., and Haas, A.L. (1982). Immunochemical analysis of the turnover of ubiquitin-protein conjugates in intact cells: Relationship to the breakdown of abnormal proteins. J. Biol. Chem. 257, 13964-13970.

31. Finley, D., Ciechanover, A., and Varshavsky, A. (1984). Thermolability of ubiquitin-activating enzyme from the mammalian cell cycle mutant ts85. Cell 37, 43-55.

32. Ciechanover, A., Finley D. and Varshavsky, A. (1984). Ubiquitin dependence of selective protein degradation demonstrated in the mammalian cell cycle mutant ts85. Cell 37, 57-66.

33. Hershko, A. and Heller, H. (1985). Occurrence of a polyubiquitin structure in ubiquitin-protein conjugates. Biochem. Biophys. Res. Common. 128, 10791086.

34. Chau, V., Tobias, J. W., Bachmair, A., Mariott, D., Ecker, D., Gonda, D. K., and Varshavsky, A. (1989). A multiubiquitin chain is confined to specific lysine in a targeted short-lived protein. Science 243, 1576-1583.

35. Hough, R., Pratt, G., and Rechsteiner, M. (1986). Ubiquitin-lysozyme conjugates. Identification and characterization of an ATP-dependent protease from rabbit reticulocyte lysates. J. Biol. Chem. 261, 2400-2408.

36. Waxman, L., Fagan, J.M., and Goldberg, A.L. (1987). Demonstration of two distinct high molecular weight proteases in rabbit reticulocytes, one of which degrades ubiquitin conjugates. J. Biol. Chem. 262, 2451-2457.

37. Heinemeyer, W., Kleinschmidt, J.A., Saidowsky, J., Escher, C., and Wolf, D.H. (1991). Proteinase yscE, the yeast proteasome/multicatalyticmultifunctional proteinase: mutants unravel its function in stress-induced proteolysis and uncover its necessity for cell survival. EMBO J. 10, 555-562.

38. Hershko, A., Heller, H., Eytan, E., Kaklij, G., and Rose, I.A. (1984). Role of $\alpha$-amino group of protein in ubiquitin-mediated protein breakdown. Proc. Natl. Acad. Sci. USA 81, 7021-7025. 
39. Reiss, Y., Kaim, D., and Hershko, A. (1988). Specificity of binding of $\mathrm{NH}_{2}-$ terminal residue of proteins to ubiquitin-protein ligase: Use of amino acid derivatives to characterize specific binding sites. J. Biol. Chem. 263, 2693-2698.

40. Ciechanover, A., Wolin, S.L., Steitz, J.A. and Lodish, H.F. (1985). Transfer RNA is an essential component of the ubiquitin and ATP-dependent proteolytic system. Proc. Natl. Acad. Sci. USA 82, 1341-1345.

41. Ferber, S., and Ciechanover, A. (1987). Role of arginine-tRNA in protein degradation by the ubiquitin pathway. Nature $326,808-811$.

42. Bachmair, A., Finley, D., and Varshavsky, A. (1986). In vivo half-life of a protein is a function of its $\mathrm{N}$-terminal residue. Science 234, 179-186.

43. Bachmair, A., and Varshavsky, A. (1989). The degradation signal in a shortlived protein. Cell 56, 1019-1032.

44. Mayer, A. Siegel, N.R., Schwartz, A.L., and Ciechanover, A. (1989). Degradation of proteins with acetylated amino termini by the ubiquitin system. Science 244, 1480-1483.

45. Hochstrasser, M., and Varshavsky, A. (1990). In vivo degradation of a transcriptional regulator: the yeast $\alpha 2$ repressor. Cell $61,697-708$.

46. Scheffner, M., Werness, B.A., Huibregtse, J.M., Levine, A.J., and Howley, P.M. (1990). The E6 oncoprotein encoded by human papillomavirus types 16 and 18 promotes the degradation of p53. Cell 63, 1129-1136.

47. Glotzer, M., Murray, A.W., and Kirschner, M.W. (1991). Cyclin is degraded by the ubiquitin pathway. Nature 349, 132-138.

48. Hershko, A., Ganoth, D., Pehrson, J., Palazzo, R.E., and Cohen, L.H. (1991). Methylated ubiquitin inhibits cyclin degradation in clam embryo extracts. J. Biol. Chem. 266, 16376-16379.

49. Ciechanover, A., DiGiuseppe, J.A., Bercovich, B., Orian, A., Richter, J.D., Schwartz, A.L. and Brodeur, G.M. (1991). Degradation of Nuclear Oncoproteins by the Ubiquitin System In Vitro. Proc. Natl. Acad. Sci. USA 88, 139-143.

50. Golab J., Stoklosa T., Czajka A., Dabrowska A., Jakobisiak M., Zagozdzon R., Wojcik C., Marczak M., and Wilk S. (2000). Synergistic antitumor effects of a selective proteasome inhibitor and TNF in mice. Anticancer Res. 20, 1717-1721.

51. Vanderlugt C.L., Rahbe S.M., Elliott P.J., Dal-Canto M.C., and Miller S.D. Treatment of established relapsing experimental autoimmune encephalomyelitis with the proteasome inhibitor PS-519 (2000). J. Autoimmun. 14, 205-211. 
52. Butz K., Denk C., Ullmann A., Scheffner M., and Hoppe-Seyler. F. (2000). Induction of apoptosis in human papillomavirus positive cancer cells by peptide aptamers targeting the viral E6 oncoprotein. Proc. Natl. Acad. Sci. USA 97, 6693-6697. 


\section{Contents}

Preface

A Ciechanover and M G Masucci

The Ubiquitin System and Some of Its Roles in Cell Cycle Control A Hershko

The Ubiquitin System and the N-End Rule Pathway

A Varshavsky

Phosphorylation-Dependent Substrate Recognition in Ubiquitin-Mediated Proteolysis

$M$ Tyers, $P$ Klein and T Pawson

The 26S Proteasome: A Supramolecular Assembly Designed for Controlled Proteolysis

W Baumeister and P Zwickl

Mechanisms and Regulation of Ubiquitin-Mediated, Limited

Processing of the NF- $\kappa \mathrm{B} \alpha$ Precursor Protein $\mathrm{p} 105$

A Ciechanover, $H$ Gonen, $B$ Bercovich, $S$ Cohen and A Orian

Regulation of Receptor Tyrosine Kinases by Ubiquitination

A Citri and Y Yarden

Regulation of p27 Degradation

$J$ Bloom and M Pagano 
Ubiquitin System-Dependent Regulation of Growth Hormone Receptor Signal Transduction and Effects of Oxidative Stress

CM Alves dos Santos and G J Strous

Inhibition of the Ubiquitin-Proteasome System by a Viral Repetitive Sequence

N P Dantuma and M G Masucci

Autosomal Recessive Juvenile Parkinsonism and the

Ubiquitin Pathway

K Tanaka, T Suzuki, T Chiba, T Kitami, Y Machida, S Sato, $N$ Hattori and Y Mizuno 